\title{
Das Röhren der Hirsche: Erste Vorlage des Conseil constitutionnel an den EuGH
}

VB verfassungsblog.de/

Franz C. Mayer , Maja Walter Mo 15 Apr 2013

Walter Scheel, Richard von Weizsäcker, Horst Köhler und Christian Wulff im Bundesverfassungsgericht? Eine eher ungewöhnliche Vorstellung hierzulande. Anders in Frankreich: Ehemalige Staatspräsidenten - derzeit Valéry Giscard d'Estaing, Jacques Chirac und Nicolas Sarkozy - haben dort von Rechts wegen auf Lebenszeit Sitz und Stimme im Conseil constitutionnel (CC, Verfassungsrat) im Montpensier-Flügel des Palais Royal in Paris. Dies ist aber nur eine der noch bestehenden Eigentümlichkeiten des CC im Vergleich zu herkömmlichen

Verfassungsgerichten. Gerade aus deutscher Sicht galt der CC lange Zeit eher als kümmerlicher Vorversuch einer „echten“ Verfassungsgerichtsbarkeit, im Wesentlichen auf eine Normentwurfskontrolle beschränkt, ohne öffentliches Verfahren, ohne Verbindung mit den Bürgern, ein Rat (Conseil) eben und kein Gericht.

Hier hat sich aber Einiges getan. Spätestens mit der Einführung eines konkreten Normenkontrollverfahrens, der "question prioritaire de constitutionnalité“ (QPC, Vorrangige Frage zur Verfassungsmäßigkeit) besteht für deutsche Verfassungshybris in dieser Hinsicht immer weniger Anlass. Seit 2010 kann jedermann, der Prozesspartei bzw. Verfahrensbeteiligter in einem Gerichtsverfahren ist, geltend machen, durch ein Gesetz in seinen von der Verfassung verbürgten Rechten oder Freiheiten verletzt zu sein. Am Ende der Weiterleitung dieser Rüge durch ein Filtersystem der Fachgerichte entscheidet der CC nach mündlicher Verhandlung über die Verfassungsmäßigkeit des beanstandeten Gesetzes.

Nachdem sich der CC im Laufe der Jahrzehnte bereits schrittweise immer mehr neben dem Staatspräsidenten (Art. 5 der französischen Verfassung) als „gardien de la Constitution“, als Hüter der Verfassung, etabliert hatte, genießt er mit seiner neuesten Kontrollbefugnis heute mehr denn je ein Ansehen als genuines Verfassungsgericht nach europäischem Modell.

Nun hat der CC das BVerfG sogar überholt, nämlich im Hinblick auf die konkrete, unter Beweis gestellte Bereitschaft zur Zusammenarbeit mit dem EuGH. Der CC hat am 4. April 2013 in einer durchaus überraschenden Wendung erstmals in seiner Geschichte den EuGH im Wege des Vorlageverfahrens nach Art. 267 AEUV angerufen (CC, déc. n²013-314P QPC, 4.4.2013 - M. Jeremy F., Absence de recours en cas d'extension des effets du mandat d'arrêt européen).

\section{Hintergrund: Ein liebestoller englischer Mathelehrer}

Einmal mehr ging es um den Europäischen Haftbefehl. Verfassungsfragen um den Europäischen Haftbefehl haben bisher schon eine Reihe von Verfassungsgerichten beschäftigt (zu nennen sind etwa Deutschland, Polen, Belgien und Spanien), eine Frequenz, die nachdenklich macht. Ausgangsfall für den Vorlagebeschluss des CC war die in den Medien europaweit ausgebreitete Liebesbeziehung eines Mathelehrers, Jeremy Forrest, zu einer seiner 15jährigen Schülerinnen in England. Die Flucht der beiden nach Frankreich im September 2012 endete mit der Festnahme des Lehrers in Bordeaux und seiner Überstellung nach Großbritannien aufgrund eines Europäischen Haftbefehls wegen Kindesentführung.

In Großbritannien wurde F. indessen zu einem sexuellen Missbrauch der nach britischem Recht Minderjährigen befragt und damit zu einem anderen Delikt als dem, wegen dem der Haftbefehl ausgestellt worden war. Hintergrund ist hier auf einer materiellen Ebene, dass das für eine mögliche Straftat des F. maßgebliche Alter der Minderjährigen in Frankreich und Großbritannien unterschiedlich festgelegt ist (15 Jahre gegenüber 16 Jahre). Der zuständige britische Richter verweigerte eine Inhaftierung aufgrund dieser Umstände, so dass die britischen Behörden einen 
Antrag auf entsprechende Erweiterung des Haftbefehls an ihre französischen Kollegen richteten. Die Chambre d'instruction de Bordeaux stimmte dieser Erweiterung zu. F. befindet sich gegenwärtig in Haft.

\section{Die europaverfassungsrechtliche Dimension}

Gegen die Erweiterung des Haftbefehls legte F. Rechtsmittel ein. Dies war aber vorliegend an sich gar nicht möglich. Deswegen veranlasste F. zugleich eine QPC zur französischen Strafprozessordnung (Code de procédure pénale, CPP), soweit diese in Art. 695-46 Abs. 4 für Fälle wie diesen Rechtsmittel ausschließt. Die beanstandete Norm war vom französischen Gesetzgeber eigens zur Umsetzung des Rahmenbeschlusses über den Europäischen Haftbefehl von 2002 erlassen worden.

F. rügte diese Bestimmung wegen Verstoßes gegen das Prinzip der Gleichheit vor dem Richter und das Recht auf effektiven Rechtsschutz (bzw. die Rechtsweggarantie).

Die verfassungsrechtliche Frage ist nun, ob der Ausschluss aller Rechtsmittel im französischen Recht bereits zwingend durch den europäischen Rahmenbeschluss vorgegeben ist. Der CC sieht sich in ständiger Rspr. nämlich nicht für zuständig an, nationale Gesetze am Maßstab der Verfassung zu überprüfen, wenn diese ihrerseits eindeutige und bedingungslose Vorgaben des Unionsrechts umsetzen. Ob dies der Fall ist, kann auch durch Vorlage der Fachgerichte geklärt werden, wenn bzw. bevor sie die (innerstaatliche) Vorlagefrage der QPC formulieren. Die verbundenen Rs. Melki und Abdeli von 2010 bestätigen, dass die Fachrichter in jedem aus ihrer Sicht geeigneten Moment des Verfahrens eine Vorlagefrage an den EuGH richten können. Ein Tätigwerden der Fachgerichte anstelle des CC entspräche der Lösung, die das BVerfG in jüngerer Zeit ausdrücklich als Zulässigkeitsfrage der konkreten Normenkontrolle nach Art. 100 GG entworfen hat und nach der alle Fachgerichte (gleich welcher Instanz) ggf. zur Vorlage an den EuGH angehalten sind, um bei einem in Frage stehenden Umsetzungsgesetz vorab zu klären, ob der nationale Gesetzgeber Spielräume ausgefüllt oder zwingende Vorgaben übernommen hat.

Im französischen Fall spielt aber auch Art. 88-2 der französischen Verfassung eine Rolle, nach der die Vorschriften zum Europäischen Haftbefehl innerstaatlich durch Gesetz und in Anwendung der europäischen Rechtsakte festgelegt werden. Mit dieser Verfassungsänderung wollte der verfassungsändernde Gesetzgeber im Jahr 2003 alle verfassungsrechtlichen Hindernisse ausräumen, die einer legislativen Umsetzung der zwingenden Vorgaben des Rahmenbeschlusses zum Europäischen Haftbefehl entgegenstehen können.

Der Rahmenbeschluss verlangt in Art. 27 Abs. 4 und 28 Abs. 3 lit. c) die Entscheidung der vollstreckenden Justizbehörde über Erweiterungsanträge „innerhalb von 30 Tagen“. Das ist kein explizites Verbot von Rechtsmitteln. Der vom CC veröffentlichte "Commentaire“ zur vorliegenden Entscheidung - eine Art Service des CC zum besseren Verständnis der traditionell knappen Entscheidungen - erläutert die sich hier stellende Auslegungsfrage. Einerseits könnte man aus dem Unterschied zwischen dem Begriff der (bloßen) „Entscheidung“ in Art. 27 und 28 und dem Terminus der „endgültigen Entscheidung“ an einer anderen Stelle des Rahmenbeschlusses (Art. 17) ableiten, dass in dieser Unterscheidung zwischen „endgültiger Entscheidung“ und „Entscheidung“ ein Hinweis darauf liegt, dass gegen letztere ein Rechtsmittel eröffnet sein muss, solche im Kontext von Art. 27 und 28 durch das europäische Recht also jedenfalls nicht untersagt sind. Hiergegen lässt sich bereits einwenden, dass - unabhängig vom Wortlaut - das Konzept von lediglich vorläufigen Entscheidungen schlicht nicht zu dem Regelungskontext Auslieferung und Überstellung passt. So einfach ist die Frage dann aber doch nicht zu lösen: Die Umsetzung der Art. 27 und 28 des Rahmenbeschlusses in den Mitgliedstaaten ist dem Commentaire zufolge nämlich höchst uneinheitlich: die Bandbreite reicht vom expliziten Ausschluss von Rechtsmitteln in Belgien über die Ausblendung der Rechtsmittelfrage in Spanien oder Italien bis zur Eröffnung von Rechtsmitteln in Großbritannien, Irland oder Malta. Das deutsche Umsetzungsgesetz (Europäisches Haftbefehlsgesetz von 2006) enthält keinen ausdrücklichen Ausschluss von Rechtsmitteln, sieht aber auch keine vor.

Der CC entschied sich vor diesem Hintergrund einer auch nach intensiven Interpretationsbemühungen offenen Auslegungsfrage des Unionsrechts zur Einleitung eines Vorabentscheidungsverfahrens, um den Regelungsgehalt 
der Art. 27 Abs. 4 und 28 Abs. 3 lit. c) des Rahmenbeschlusses durch den dafür zuständigen EuGH bestimmen zu lassen.

\section{Die erste Vorlage des CC}

Dies ist die erste Vorlagefrage des CC an den EuGH überhaupt. Der CC hatte bisher unter Verweis auf die ihm im Rahmen der präventiven Normenkontrolle nach Art. 61 Abs. 3 S. 1 der Verfassung gesetzte Entscheidungsfrist von einem Monat keine eigene Vorlagebereitschaft erkennen lassen, sondern auf die Fachgerichte verwiesen. Dies betraf Entscheidungen zum französischen Identitätsvorbehalt, welcher nach der Rspr. des CC der verfassungsrechtlichen Verpflichtung zur Richtlinienumsetzung nach Art. 88-1 der Verfassung entgegengehalten werden kann.

Vorlageverfahren dauern durchschnittlich knapp 16 Monate. Dennoch war der Verweis des CC auf seine eigenen knappen Entscheidungsfristen nie restlos überzeugend, schon weil über das Eilverfahren vor dem EuGH eine Entscheidung innerhalb kürzester Zeit möglich ist. Ferner ist unter dem Aspekt der Vorrangigkeit des Europarechts auch denkbar, dass die nationale Entscheidungsfrist durch Vorlage an den EuGH unterbrochen wird.

Es war zu erwarten, dass auch bei der neuen QPC die Verfahrensdauer als Hinderungsgrund für eine Vorlage vorgeschoben werden würde, denn auch bei diesem Verfahren bestehen relativ kurze Fristen für alle beteiligten Gerichte: Der CC muss ab seiner Befassung mit einer QPC binnen drei Monaten entscheiden. Dass der CC nun einen anderen Weg geht, überrascht.

Im vorliegenden Fall erleichtert freilich der durch den Vertrag von Lissabon eingefügte Art. 267 Abs. 4 AEUV die Dinge: Bei Vorlagefragen an den EuGH anlässlich eines schwebenden Verfahrens, das - wie hier - eine inhaftierte Person betrifft, ordnet das Unionsrecht bereits eine zügige Entscheidung an. Der CC hat entsprechend ein Eilverfahren i.S.d. Art. 267 Abs. 4 AEUV sowie gemäß Art. 23a der EuGH-Satzung (Protokoll Nr. 3 zum AEUV) beantragt. Bei dieser Art von Vorlageverfahren ist eine Entscheidung des EuGH in etwa 2 Monaten zu erwarten. Eine mögliche Überschreitung der innerstaatlichen Drei-Monats-Frist wird dabei vom CC erkannt, aber nunmehr in Kauf genommen. Insofern werden hier zwei schon bisher mögliche Lösungen des Fristenproblems kombiniert: Zwischen den Zeilen erkennt der CC an, dass das Vorabentscheidungsverfahren - und damit der Vorrang des Unionsrechts - die verfassungsrechtlichen Fristen suspendieren kann.

Je nachdem, wie schnell der EuGH nun im Frühjahr 2013 die Vorlagefrage beantwortet, könnte die Entscheidung eine Tür dahingehend geöffnet haben, vom bisherigen Fristenargument der Identitätsrechtsprechung womöglich auch in Fällen der (zeitlich strengeren) a priori-Kontrolle von Normentwürfen Abstand zu nehmen.

\section{Das Röhren der Hirsche - Konkurrenz und Konfrontation}

Warum der CC nun recht überraschend den Weg der Vorlage zum EuGH beschreitet, mag auch mit einer Veränderung seiner Zusammensetzung erklärbar sein. Der eingangs erwähnte Neuzugang im CC, der vormalige Staatspräsident Sarkozy, spielt dabei keine Rolle, er hat bei der Entscheidung vom 4. April 2013 gar nicht mitgewirkt. Es sind aber zwei der ordentlichen Mitglieder des CC kürzlich turnusmäßig ersetzt worden.

Die Vorlagen nationaler Höchst- und Verfassungsgerichte stehen in einem allgemeineren Zusammenhang. Mittlerweile haben fast alle Höchst- und Verfassungsgerichte der EU mindestens einmal den Weg des Vorlageverfahrens beschritten. Auf der Liste der vorlegenden Gerichte finden sich das traditionsreiche britische House of Lords (und in jüngerer Zeit auch der neue Supreme Court) wie der durchaus europakritische dänische Højesteret mit regelmäßigen Vorlagen ebenso wie der österreichische VfGH oder der belgische Verfassungsgerichtshof, und neuerdings auch das spanische und italienische Verfassungsgericht. Die Nichtvorlagepraxis des BVerfG erscheint immer mehr als deutscher Verfassungssonderweg (bisher noch gefolgt durch die Verfassungsgerichte in Polen und Tschechien). Zwar hat das BVerfG schon 1986 für sich anerkannt, an Art. 267 AEUV grundsätzlich gebunden zu sein (ohne indessen auch eine entsprechende Verpflichtung deutlich 
ausgesprochen zu haben). In Medienäußerungen von Richtern wird auch immer wieder bedeutet, man habe kein grundsätzliches Problem mit dem EuGH und einer Vorlage zum EuGH. Danach wäre eine Vorlage des BVerfG nur eine Frage der Zeit und eines passenden Falles... Noch offene Fragen um die europarechtliche Zulässigkeit von Sekundärmarktankäufen der EZB könnten im noch anhängigen Verfahren in Sachen ESM und Fiskalpakt hier schon bald zum Lackmustest werden.

Der CC jedenfalls beweist, dass es sogar unter ungünstigen nationalen Verfassungsbedingungen - hier: knappe Entscheidungsfristen - einen Weg zum EuGH gibt und dass es keines besonders passenden Falles und einer entsprechenden Vorlageinszenierung bedarf. Die Vorlageentscheidung des CC wirkt (sicherlich auch aufgrund des typischen knappen Duktus) sehr unprätentiös. Sie ist ein wichtiges Zeichen gegenüber dem EuGH, das wie die Rechtsprechung um die Rs. Melki und Abdeli Kooperation und Offenheit zwischen den Ebenen signalisiert. Die Eurokrise zeigt, wie dünn und fragil der Firnis der Rechtsgemeinschaft über den Antagonismen der Vergangenheit und den Egoismen in Europa auch nach sechs Jahrzehnten europäischer Integration noch immer ist. Dass die Höchstgerichte in der EU durch konstruktives Zusammenwirken hier einen Gegenakzent setzen, ist von besonderer Dringlichkeit.

Der unaufgeregte Stil der Entscheidung des CC vom 4. April 2013 unterscheidet sich vom Platzhirschröhren in Verfassungsgerichtsentscheidungen und jenseits davon in Richterinterviews und -vorträgen, wie es gelegentlich aus Karlsruhe oder Brno und anderswo erklingt. Im europäischen Verfassungsverbund wie auch sonst bedeutet Dialog nicht, dass zwei gleichzeitig und möglichst laut sprechen. Es geht vielmehr um einen echten Austausch zwischen den Gerichten und eine gemeinsame Orientierung an gemeinsam durch die Gerichte zu ermittelnden und zu sichernden Verfassungsgewährleistungen.

\section{LICENSED UNDER CC BY NC ND}

SUGGESTED CITATION Mayer, Franz C.; Walter, Maja: Das Röhren der Hirsche: Erste Vorlage des Conseil constitutionnel an den EuGH, VerfBlog, 2013/4/15, http://verfassungsblog.de/das-rohren-der-hirsche-erste-vorlagedes-conseil-constitutionnel-an-den-eugh/. 